\title{
Area law and vacuum reordering in harmonic networks
}

\author{
A. Riera and J. I. Latorre \\ Dept. d'Estructura i Constituents de la Matèria, Univ. Barcelona, 08028, Barcelona, Spain.
}

(Dated: 7th May 2018)

\begin{abstract}
We review a number of ideas related to area law scaling of the geometric entropy from the point of view of condensed matter, quantum field theory and quantum information. An explicit computation in arbitrary dimensions of the geometric entropy of the ground state of a discretized scalar free field theory shows the expected area law result. In this case, area law scaling is a manifestation of a deeper reordering of the vacuum produced by majorization relations. Furthermore, the explicit control on all the eigenvalues of the reduced density matrix allows for a verification of entropy loss along the renormalization group trajectory driven by the mass term. A further result of our computation shows that single-copy entanglement also obeys area law scaling, majorization relations and decreases along renormalization group flows.
\end{abstract}

PACS numbers: 03.75.Ss, 03.75.Lm, 03.75.Kk

\section{INTRODUCTION}

The amount of entanglement present in a quantum state is of fundamental relevance to determine how hard it is to simulate it by classical means. It is generally argued that a highly entangled quantum state carries a huge superposition of product states that cannot be handled on a classical computer. Yet, this statement must be made precise, since a small amount of entanglement can indeed be simulated efficiently. The relevant precise question is, thus, how much entanglement can be efficiently simulated classically.

This abstract question should at least be clarified when considering relevant physical systems. Can the amount of entanglement present in a two-dimensional lattice of harmonic oscillators be efficiently represented in a classical computer? Although the answer to this question is not yet settled, qualitative progress has been recently achieved. One of the ingredients essential to this discussion is the area law for the geometric entropy and the representation of quantum states by projected entangled pairs.

A important related problem is to understand how entanglement varies along renormalization group (RG) trajectories. We shall bring growing evidence for the idea that RG flows entail a loss of entanglement. This entanglement loss will be shown compatible with area law scaling of the entropy.

We organize the contents of this paper by first reviewing a number of previous results on area law scaling of ground state entropy in different systems using the language of condensed matter, quantum field theory and quantum information theory. We shall then present a computation of entanglement entropy on a discretized bosonic free field theory in arbitrary dimensions. This gives us control on the eigenvalues of the reduced density matrix on a subsystem which, in turn, allows for a discussion of majorization relations obeyed by the reduced density matrix of the system. We extend this discussion to the single-copy entanglement measure. RG loss of entanglement is also verified in detail for arbitrary dimension networks of harmonic oscillators.

\section{A BRIEF REVIEW OF THE AREA LAW}

\section{A. Measures of entanglement for many-body systems}

An arbitrary quantum state of e. $g$. $N$ spins is, in general, highly entangled. To quantify such a statement we can use various figures of merit. For instance, concurrence [1] is easy to compute and detects some pairwise entanglement though it cannot scan correlations throughout the system. An appropriate and widely used candidate to quantify entanglement is the von Neumann entropy of the reduced density matrix of the state under analysis, when only a subset of degrees of freedom is retained. To be precise, let us consider a quantum state made out of $N$ qubits $|\psi\rangle \in\left(C^{2}\right)^{\otimes N}$ and its density matrix of a $\rho \equiv|\psi\rangle\langle\psi|$. Next, we consider the reduced density matrix of a subset of qubits denoted by $A, \rho_{A} \equiv \operatorname{Tr}_{\bar{A}} \rho$ where all qubits but those belonging to the set $A$ are traced out. The von Neumann entropy of the reduced density matrix is then defined as

$$
S\left(\rho_{A}\right)=-\operatorname{Tr} \rho_{A} \log \rho_{A} .
$$

The entropy is often referred to as entanglement entropy.

In general a set of particles will be distributed randomly over space. Entanglement entropy can be computed for all sorts of partitions of the system, yielding information about the quantum correlations among the chosen subparts. A particular and extremely relevant class of physical systems are those made of local quantum degrees of freedom which are arranged in chains or, more generally, in networks. For such systems it is natural to analyze their entanglement by studying geometrical partitions, that is, computing the entanglement entropy between a set of contiguous qubits versus the rest of the system. We shall referred to this particular case of entanglement entropy [2] as geometric entropy [3] (also called fine grained entropy in [4]).

The appearance of scaling of the geometric entropy with the size of the sub-system under consideration has been shown to be related to quantum phase transitions in one-dimensional systems, further reflecting the universality class corresponding to the specific phase transition under consideration [3, 5] (see also [6]). Broadly speaking, a large entropy is related to 
the presence of long distance correlations, whereas a small entropy is expected in the presence of a finite correlation length. The precise scaling of geometric entropy does eventually determine the limits for today's efficient simulation of a physical quantum system on a classical computer.

There are many other ways to quantify entanglement. The von Neumann entropy we have chosen as our central figure of merit has an asymptotic operational meaning. Given infinitely many copies of a bipartite quantum state, it quantifies how many EPR pairs can be obtained using local operations and classical communication. A different measure of entanglement can be associated with the analysis of entanglement on a single-copy of the quantum system. This single-copy entanglement [7, 8, 9, 10] can be defined as

$$
E_{1}\left(\rho_{A}\right)=-\log \rho_{A}^{(1)}
$$

where $\rho_{A}^{(1)}$ is the maximum eigenvalue of $\rho_{A}$. This quantity provides the amount of maximal entanglement that can be extracted from a single copy of a state by means of local operations and classical communication. As we shall see later on, the von Neumann entropy and the single-copy entanglement appear to be deeply related in any number of dimensions.

\section{B. Volume vs. area law}

Random states are known to carry large entanglement. To be precise, let us consider a random infinite system o qubits. On average, the density matrix for a random subset of $N$ qubits carries maximum von Neumann entropy,

$$
S\left(\rho_{N}\right) \sim N .
$$

This result [11] shows that the entropy of random states grows as the number of particles included in the subset. This is referred to as a volume law scaling. An arbitrary state uses the maximum possible superposition of the basis elements with no symmetry whatsoever among their coefficients. Its efficient representation by classical means appears certainly difficult.

Physical theories create entanglement through interactions, which are typically local. Thus, e.g. the ground state of a sensible physical Hamiltonian is not a random state. It is natural to expect a low amount of entropy since local interactions will entangle the non-contiguous degrees of freedom in a somewhat sequential way. We may encounter local intense entanglement that dilutes at long distance. This is precisely the structure of standard quantum theories, with correlations that decay with a power law at phase transitions and with an exponential law away from them. It is then reasonable to ask what is the limit of efficient simulability in terms of the entanglement present in a given state.

In many physical theories, local degrees of freedom are arranged in a specific geometrical way as mentioned previously. We may have quantum systems defined on spin chains, networks or, in general, $D$-dimensional lattices. Those systems may have a continuum limit described by a quantum field theory or, alternatively, may be devised as quantum simulators, a preview of quantum computers. We may then discuss the amount of geometrical entanglement present on the system from three complementary points of view: condensed matter, quantum field theory and quantum information.

As we shall see, the basic ingredient of locality of interactions suggests that entropy for a geometrical region should be dominated by the entanglement present on the surface separating it from the rest of the system. To be precise, consider an infinite $D$-dimensional lattice where we assign part $A$ to an inner hypercube of size $L, N=L^{D}$, and part $B$ to the outside. Locality seems to suggest

$$
S\left(\rho_{L}\right) \sim L^{D-1} \sim N^{\frac{D-1}{D}} .
$$

This behavior is commonly referred to as area law scaling for the geometric entropy. Let us note that one-dimensional quantum systems correspond to a well understood limiting case for the above formula, where the power law turns out to be substituted with a logarithmic scaling at phase transitions, that is

$$
S\left(\rho_{L}\right) \sim \log L,
$$

and saturates away from them

$$
S\left(\rho_{L}\right)<\text { constant } \quad, \forall L \quad,
$$

as shown in Ref. [5, 12, 13] These results are deeply connected to conformal symmetry and control the classical simulability of the system.

Recent evidence hints at a log violation of the area law in some two-dimensional systems made with anticommuting variables [14, 15, 16, 17]. To be precise, some of these models display an entropy scaling law of the type

$$
S\left(\rho_{L}\right) \sim L^{D-1} \log L .
$$

It is unclear whether such systems support a limiting quantum field theory description in the continuum limit.

It is important to make a general remark concerning the different approaches to the computation of entanglement in quantum systems. Let us note that discretized quantum systems allow for uncontroversial computations of the entropy. This is not the case of quantum field theories, where regularization and renormalization are needed since the number of degrees of freedom is formally unbounded. In such a framework, the adimensional entropy requires the appearance of some short-distance regulator $\epsilon$

$$
S\left(\rho_{L}\right) \sim\left(\frac{L}{\epsilon}\right)^{D-1}
$$

which entails the necessary discussion of its renormalization and its observability. Let us just mention here that the coefficient of the area law is universal for $D=1$ systems whereas remains scheme-dependent in higher dimensions.

The problem turns extremely subtle in the case of gravity, where the geometry of space-time is dynamical and the way to compute for a black hole the Bekenstein area law pre-factor from first principles is far from clear[18, 19, 20]. Recent progress on the side of AdS/CFT correspondence seems to link entanglement entropy in a quantum field theory living on the boundary to the black-hole entropy of the bulk [19, 20]. 


\section{Locality and PEPS}

The basic heuristic argument for an area law scaling of entropy for the ground state of physical systems is rooted in the locality of the interactions. Steps to make this argument quantitative have been made in Refs. [21, 22, 23, 24, 25, 26].

A local Hamiltonian tends to entangle nearest neighbors. Long-distance entanglement emerges as a coherent combination of local interactions. The correctness of this argument would imply that the reduced entropy of a geometric bipartition of a system will get its main contribution from the entanglement between degrees of freedom at opposite sites of the boundary that separates the regions. This, in turn, implies an area-law scaling. Let us note that such a naive argument works in any dimension and does not depend on the correlation length present in the system. Area law would emerge from locality, whatever the mass-gap is. We shall discuss the limitations of this argument shortly.

This argument needs a clear formulation and verification. Although we lack definite answers about the necessary and sufficient conditions a Hamiltonian must obey to produce a ground state with area law entropy, some progress has been achieved using one-dimensional Matrix Product States (MPS) and their generalization to higher dimensions, Projected Entangled Pair States (PEPS). We first consider a onedimensional system with open boundary conditions described by a MPS

$$
|\psi\rangle=\sum\left(A_{\alpha_{1}}^{i_{1}} A_{\alpha_{1} \alpha_{2}}^{i_{2}} \ldots A_{\alpha_{n-1}}^{i_{n}}\right)\left|i_{1} \ldots i_{n}\right\rangle
$$

where the sum extends to $i_{1}, \ldots, i_{n}=1, \ldots, d$, which are physical indices attached to local Hilbert spaces, and $\alpha_{1}, \ldots, \alpha_{n-1}=1, \ldots \chi$, which are ancillae indices. The tensors $A_{\alpha \beta}^{i}$ can be viewed as projectors from the ancillae indices to a physical one. This representation provides the basis for the density matrix renormalization group technique.

The generalization of the MPS construction to higher dimensional networks carries the name of PEPS. In a $D$ dimensional network, where ancillae degrees of freedom are linked to their nearest neighbors, the role of the MPS projector is taken by a tensor of the form

$$
A_{\alpha \underset{\delta}{\gamma} \beta}^{a}
$$

where the physical indices span a $D$-dimensional lattice and ancillae run from 1 to $\chi$. Again, the role of each tensor $A$ is to project maximally entangled pairs connecting local neighbors onto a physical local space. Entanglement is thus carried by the links connecting ancillae. Each entangled pair, that is, each sum over one ancilla index hides a connecting bond of the type $\sum_{\alpha=1}^{\chi} \frac{1}{\sqrt{\chi}}|\alpha \alpha\rangle$. If one of the two ancillae in the bond is traced out, the entropy for the remaining ancilla is $S=\log \chi$.

We are now in a position to present the argument in Ref. [21] showing that finite $\chi$ PEPS entail area law scaling for the entropy. Let us assume that the ground state of a quantum system is described by a PEPS with finite $\chi$. It follows that the entropy of a subpart of the system is bounded by the number of bonds which are cut by the separating surface times the entropy per broken bond. This amounts to an area law

$$
S\left(\rho_{A}\right) \leq(\# \text { cut bonds }) \log \chi \sim \text { Area } \log \chi
$$

A violation of the area law within the PEPS representation requires infinite-dimensional ancillae.

We should again distinguish the one-dimensional case, where the ground state of infinite critical systems are known to carry logarithmic entropy [5],

$$
S\left(\rho_{L}\right) \sim \frac{c}{3} \log \frac{L}{a}
$$

where $a$ is the lattice spacing and $c$ the central charge that characterizes the universality class of the phase transition. Yet, the boundary of a one-dimensional block is made by two single points. Such a state with logarithmic entropy cannot be represented using finite dimensional MPS and we must resort to arbitrarily large $\chi$. This limitation is at the heart of the problems that the DMRG technique encounters when applied to quantum phase transitions. On the other hand, the entropy is bounded away from critical points and MPS provide an efficient way to represent the system. MPS states with finite $\chi$ are often referred to as finitely correlated states.

Coming back to higher dimensions, it is then a major issue to establish whether finite $\chi$ PEPS can describe faithfully the ground state of physical systems. The fact that PEPS with finite $\chi$ can incorporate an area law is appealing. Recently, a particular class of finite PEPS has been constructed that display polynomial decay laws, that is long range correlation [26]. These PEPS are also shown to describe ground states of frustration-free Hamiltonians and such states can approximate exponentially well any finitely correlated state. It is still unclear whether the ground states of standard quantum systems fall into this description or, alternatively, they need infinite $\chi$. This may set apart what is efficiently simulable from what is not.

\section{Renormalization group transformations on MPS and PEPS and the support for an area law}

We have argued that one-dimensional finite $\chi$ MPS can support a maximum amount of entropy independent of the size of the system and that, in contradistinction, finite D-dimensional PEPS can accommodate an area law. Let us give an independent quantitative argument for this statement.

Consider a renormalization group transformation of a MPS state with constant $A$ defined by the coarse graining of two sites [27]

$$
A_{\alpha \beta}^{i} A_{\beta \gamma}^{j} \equiv \tilde{A}_{\alpha \gamma}^{i j}=\sum_{l=1}^{\min (d 2, \chi 2)} \lambda_{l} U_{l}^{(i j)} V_{\alpha \gamma}^{l}
$$

where we have decomposed the product of two adjacent matrices using a singular value decomposition. We can understand the unitary matrix $U$ as a change of basis on the new 
coarsed degree of freedom and construct a new MPS with $A_{\alpha \gamma}^{\prime l}=\lambda_{l} V_{\alpha \gamma}^{l}$. Therefore, the ancillae indices close under such operation whereas the physical index grows. Upon iteration of this operation, the range of the physical index will reach a maximum value $\chi 2$ and will get locked to that value. This is the magic of one dimension. The long-distance properties of the system are completely described by a single square effective matrix! Entropy is then bounded.

The analogous argument in two-dimensional systems follows a slightly different path. The coarse graining step reads

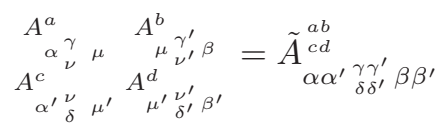

$$
\begin{aligned}
& =\sum_{l=1}^{\min \left(d 4,\left(\chi^{4}\right) 2\right)} \lambda_{l} U_{l}^{a b} V_{\alpha \alpha_{\delta \delta^{\prime}}^{c d} \beta \beta^{\prime}}^{l}
\end{aligned}
$$

As before, we can absorb the global $U$ as a change of the local coarse-grained basis and assign a new PEPS to $\lambda V$. Note the different growth of indices. On the one hand, physical indices merge in groups of four and would naively need a volume law increase, $d 4$. On the other hand, the ancillae rank increase from $\chi 4$ to $(\chi 4) 2$, that is, it follows an area law. Given that the singular value decomposition will be locked by the smallest dimension of the two above, the area law will define the rank of the tensor that contains the effective long distance description of the model. The argument generalizes to $D$ dimensions where the PEPS $A_{\alpha_{1}, \ldots, \alpha_{2 D}}^{i}$ with a physical index $i=1, \ldots, d$ and ancillae indices $\alpha_{1}, \ldots, \alpha_{2 D}=1, \ldots, \chi$. A renormalization group transformation of this PEPS makes the new collective physical index to run $i^{\prime}=1, \ldots, d^{2^{D}}$, that is, with a volume law, and the new collective ancillae $\alpha_{1}^{\prime}, \ldots, \alpha_{2 D}^{\prime}=1, \ldots, \chi^{2^{D-1}}$, that is, as an area law. The singular value decomposition makes all the long-distance properties of the state to be contained in an effective PEPS with a number of degrees of freedom that grows with just an area law. The rank of the effective PEPS is $\log \chi_{\text {eff }}=2^{D-1} \log \chi$. From this simple argument, it follows that PEPS can support an area law scaling for the geometrical entropy.

\section{E. Some explicit examples of area law}

There is an extensive literature on computations of the entropy for particular cases that cannot be faithfully summarized here.

One-dimensional spin systems (e.g. quantum Ising model, XX model and Heisenberg model) obey a logarithmic scaling at the critical point [5, 12, 13, 28, 29, 30]. Away from the quantum phase transition point, the entropy gets saturated. This explicit computation falls into the universal scaling predicted by conformal invariance. This result has been further verified and extended to many other quantum systems in one dimension. I

The literature on computations of entanglement entropy in higher dimensional systems is far less extensive due to the difficulty to produce explicit results. The first analysis of the entanglement entropy in two- and three-dimensional systems

\begin{tabular}{cc}
\hline Spin chains away from criticality & $S \sim$ constant \\
Critical spin chains & $S \sim \log N$ \\
D-dimensional harmonic networks & $S \sim N^{\frac{D-1}{D}}$ \\
NP-complete problems & $S \sim N$ \\
\hline
\end{tabular}

were done in discretized approaches to quantum field theory [2, 4, 31]. Further analysis showed that the entanglement entropy is related to the trace anomaly in curved space times giving an explicit relation between the actual results for free fermions and free bosons [32].

Rigorous computations in discretized harmonic networks proved no departure from the area law [23, 33]. Further analysis of entanglement entropy on higher dimensional networks has been done in Refs. [34, 35, 36].

\section{F. Exceptions to the area law}

We have argued that the area law is deeply connected to locality of interactions. It is, therefore, reasonable to expect violations of such scaling in models with non-local interactions. This is the generic case of a quantum computation of an NP-complete problem. It has been numerically verified that this is the case when an adiabatic quantum computation is applied to the NP-complete Exact Cover problem, a variant of the 3-SAT problem. Along the computation, the ground state becomes maximally entangled, that is its entropy scales as the volume of the system [37, 38]. A physical quantum computer will definitely need to face the challenge of maintaining those huge fine-tuned superpositions of states.

Locality of interactions is not the only ingredient that controls entropy. Entropy is related to the eigenvalues of the Schmidt decomposition of a system in two parts. If the subsystems retain a lot of symmetry, the sub Hilbert spaces organize themselves in representations of the symmetry group. This entails a reduction of the Schmidt number of the above decomposition, that is, a lower entropy. Such a counter mechanism to reduce the entropy in highly connected systems has been explicitely checked in the case of the Lipkin-Meshkov model which is defined by a spin system fully and symmetrically connected. Although it is tempting to argue that the system is infinite-dimensional (the geometry of the Hamiltonian corresponds to a simplex of $N \rightarrow \infty$ vertices), the entropy scales only logarithmically, which is the actual bound for symmetric spaces [39]. This logarithmic scaling of the entropy follows the one-dimensional log law, which might just be an accident.

It should not come as a surprise that slightly entangled states that do not correspond to an eigenstate of a given Hamiltonian dynamically evolve to highly entangled states under its action. This has been analyzed in Refs. [40, 41] even for simple Hamiltonians like the quantum Ising chain. No area law is expected for slightly entangled random states when they are evolved with local Hamiltonians.

As mentioned previously, a case of non-trivial violation of 
the area law was first considered in [14] and then analyzed in [15, 17]. Some two-dimensional systems with anticommuting variables were found to display a $\log$ correction to the area law, that is, $S \sim L \log L$. On the other hand, some previous computations for free Dirac fermions seem to produce no area-law violation [42, 44, 45] in any number of dimensions. This issue deserves further investigation. Finally let us mention that in the computation of quantum corrections to the entropy of a black hole, logarithmic corrections have also been obtained [43].

\section{G. Physical and computational meaning of an area law}

We can attach physical meaning to an area law scaling of entropy in different but related ways. We may argue that entropy is a measure of surprise due to quantum correlations and that a state that obeys an area law carries less correlations than a random state. As the size of the inner block increases, we only get a reduced amount of surprise, compare to the maximum possible, when discovering that our block was correlated to the exterior. It is then arguable that the theory that has produced such a state may accept a simpler description. In some sense, this argument is implicit in the holographic description of some quantum systems.

From a computational point of view, low entropy means small quantum correlations, that is, small entanglement. It is known that states that are only slightly entangled can be efficiently simulated by classical means [46]. A fundamental question is thus formulated: what entropy growth law can be efficiently simulated by a classical computer?

So far, this question can only be answered partially. In one dimension, $D=1$, quantum critical phenomena show a logarithmic scaling which cannot be reproduced using finite MPS techniques. Formally, the simulation remain efficient in the sense that to reproduce critical behavior we need $\chi$ to be polynomial in $L$. This, though, produces an obvious practical computational slowing down and limitation. A new promising idea to represent a quantum state with a different and nonlocal tensor structure has been proposed in Ref. [47] with the name of multi-scale entanglement renormalization ansatz (MERA). The basic idea is to substitute a linear MPS representation with a RG-inspired construction that also identifies the key use of disentangling operations for blocks before proceeding to a coarsed description.

The question in two dimensions has been addressed in [21] in a sequential way. A PEPS is taken as lines of spins that are collected into effective degrees of freedom which are further treated in a MPS manner.

\section{AREA LAW IN $D$ DIMENSIONS}

\section{A. The Hamiltonian of a scalar field in $D$ dimensions}

Let us consider the theory of a set of harmonic oscillators in $D$ dimensions which is expected to verify area law scaling of the entropy. A number of non-trivial issues can be discussed in this explicit example. First, we shall analyze the regularized version of a scalar free field theory in order to get its reduced density matrix when an inner geometrical ball is integrated out. Its eigenvalues can, then, be used to compute the geometrical entropy that will scale as dictated by the area law. Second, we can compare the behavior of the entropy to the one of the single-copy entanglement. Third, we can analyze whether area law scaling is backed by a deeper sense of order, namely majorization theory.

Our computation will generalize the one presented in Ref. [2] to $D$ dimensions. Let us consider the Klein-Gordon Hamiltonian

$$
H=\frac{1}{2} \int \mathrm{d}^{D} x\left(\pi^{2}(\vec{x})+|\nabla \phi(\vec{x})|^{2}+\mu^{2}|\phi(\vec{x})|^{2}\right),
$$

where $\pi(x)$ is the canonical momentum associated to the scalar field $\phi(x)$ of mass $\mu$. The $D$-dimensional Laplacian reads

$$
\Delta \phi=\frac{1}{r^{D-1}} \frac{\partial}{\partial r}\left(r^{D-1} \frac{\partial \phi}{\partial r}\right)+\frac{1}{r^{2}} \hat{L}^{2} \phi
$$

where $r=|\vec{x}|$ and $\hat{L}^{2}$ is the total angular momentum operator in $D$ dimensions. It is convenient to introduce the real spherical harmonic functions $Z_{l\{m\}}$, which are eigenfunctions of $\hat{L}^{2}$ with eigenvalues $l(l+D-2)$. The set of numbers $\{m\}$ stand for other Casimir and component labels in the group $S O(D)$. We now project the angular part of the scalar fields $\pi$ and $\phi$,

$$
\begin{aligned}
& \pi_{l\{m\}}(r)=r^{\frac{D-1}{2}} \int \mathrm{d}^{D} x Z_{l\{m\}}\left(\theta_{1}, \cdots, \theta_{D-2}, \varphi\right) \pi(\vec{x}) \\
& \phi_{l\{m\}}(r)=r^{\frac{D-1}{2}} \int \mathrm{d}^{D} x Z_{l\{m\}}\left(\theta_{1}, \cdots, \theta_{D-2}, \varphi\right) \phi(\vec{x}),
\end{aligned}
$$

where $r, \theta_{1}, \cdots, \theta_{D-2}$ and $\varphi$ define the spherical coordinates in $D$ dimensions. The Hamiltonian now reads

$$
H=\sum_{l\{m\}} H_{l\{m\}}
$$

where,

$$
\begin{aligned}
H_{l\{m\}} & =\frac{1}{2} \int \mathrm{d} r\left(\pi_{l\{m\}}^{2}(r)+r^{D-1}\left(\frac{\partial}{\partial r}\left(\frac{\phi_{l\{m\}}(r)}{r^{\frac{D-1}{2}}}\right)\right)^{2}\right. \\
& \left.+\left(\frac{l(l+D-2)}{r^{2}}+\mu^{2}\right) \phi_{l\{m\}}^{2}(r)\right) .
\end{aligned}
$$

An ultraviolet regularization of the radial coordinate in the above Hamiltonian will transform the scalar field theory into 
a chain of coupled harmonic oscillators. This is achieved by discretizing the continuous radial coordinate $r$ into a lattice of $N$ discrete points spaced by a distance $a$,

$$
\begin{aligned}
H_{l\{m\}} & =\frac{1}{2 a} \sum_{j=1}^{N}\left(\pi_{l\{m\}, j}^{2}\right. \\
& +\left(j+\frac{1}{2}\right)^{D-1}\left(\frac{\phi_{l\{m\}, j+1}}{(j+1)^{\frac{D-1}{2}}}-\frac{\phi_{l\{m\}, j}}{j^{\frac{D-1}{2}}}\right)^{2} \\
& \left.+\left(\frac{l(l+D-2)}{j^{2}}+\mu^{2}\right) \phi_{l\{m\}, j}^{2}(x)\right) .
\end{aligned}
$$

The size of the system is $L=(N+1) a$, where $a$ and $L$ act as an ultraviolet and infrared cutoff respectively. We can compare this expression with the Hamiltonian of an open chain of $N$ coupled harmonic oscillators,

$$
H=\frac{1}{2} \sum_{i=1}^{N} p_{i}^{2}+\frac{1}{2} \sum_{i, j=1}^{N} x_{i} K_{i j} x_{j}
$$

and identify $K_{i j}$ as

$$
\begin{aligned}
K_{i j} & =\left(\frac{l(l+D-2)}{j^{2}}+\mu^{2}\right) \delta_{i j} \\
& +\left(1-\frac{1}{2 j}\right)^{D-1} \theta\left(j-\frac{3}{2}\right) \delta_{i j} \\
& +\left(1+\frac{1}{2 j}\right)^{D-1} \theta\left(N-\frac{1}{2}-j\right) \delta_{i j} \\
& +\left(\frac{j+\frac{1}{2}}{\sqrt{j(j+1)}}\right)^{D-1} \delta_{i, j+1}+\left(\frac{i+\frac{1}{2}}{\sqrt{i(i+1)}}\right)^{D-1} \delta_{i+1, j},
\end{aligned}
$$

where $\theta$ is the step function.

\section{B. Geometric entropy and single-copy entanglement}

We now proceed to trace out an inner geometric ball around the origin to obtain the reduced density matrix of the ground state of the system on the exterior of that ball. Following similar steps as in [2] we define $\Omega$ as the square root of $K$, that is $K=\Omega 2$. The gaussian ground state of the system can be expressed as,

$$
\psi_{0}\left(x_{1}, \ldots, x_{N}\right)=\pi^{-N / 4}(\operatorname{det} \Omega)^{1 / 4} e^{-\frac{x^{T} \cdot \Omega \cdot x}{2}},
$$

where $x \equiv\left(x_{1}, \ldots, x_{N}\right)$. We construct the density matrix $\rho_{\text {out }}$ by tracing over the inner $n$ oscillators,

$$
\rho_{\text {out }}\left(x, x^{\prime}\right) \sim e^{-\frac{1}{2}\left(x^{T} \cdot \gamma \cdot x+x^{\prime T} \cdot \gamma \cdot x^{\prime}\right)+x^{T} \cdot \beta \cdot x^{\prime}},
$$

where $\beta$ and $\gamma$ are defined by

$$
\begin{aligned}
\beta & \equiv \frac{1}{2} B^{T} A^{-1} B \\
\gamma & \equiv C-\beta
\end{aligned}
$$

and $A=\Omega(1 \div n, 1 \div n), B=\Omega(1 \div n, n+1 \div N)$ and $C=$ $\Omega(n+1 \div N, n+1 \div N)$ are sub-matrices of $\Omega$.

We proceed with the diagonalization of this structure rotating and rescaling the variables $x=V^{T} \gamma_{D}^{-1 / 2} y$ where $\gamma=V^{T} \gamma_{D} V$ and $\gamma_{D}$ is diagonal. Using this transformation, $\gamma$ becomes identity, $\beta \rightarrow \beta^{\prime}=\gamma_{D}^{-1 / 2} V \beta V^{T} \gamma_{D}^{-1 / 2}$ and the density matrix reads

$$
\rho_{\text {out }}\left(y, y^{\prime}\right) \sim e^{-\frac{1}{2}\left(y^{2}+y^{\prime 2}\right)+y^{T} \cdot \beta^{\prime} \cdot y^{\prime}} .
$$

If we do the appropriate change of coordinates $y=W \cdot z$ (where $W$ is an orthogonal matrix) such that $W^{T} \cdot \beta^{\prime} \cdot W$ becomes diagonal with eigenvalues $\beta_{i}^{\prime}$, we get $\rho_{\text {out }}$ as a tensor product of the two coupled harmonic oscillators density matrices,

$$
\rho_{\text {out }}\left(z, z^{\prime}\right) \sim \prod_{i=1}^{N-n} e^{-\frac{1}{2}\left(z_{i}^{2}+z_{i}^{\prime 2}\right)+\beta_{i}^{\prime} z_{i} z_{i}^{\prime}} .
$$

We can now compute the entropy associated to the reduced density matrix $\rho_{\text {out }}$. This entropy can be expressed as a sum over contributions coming from each term in the reduced density matrix tensor product structure,

$$
S_{l\{m\}}=\sum_{i=1}^{N-n} S_{l\{m\}, i}\left(\xi_{i}\right),
$$

where

$$
S_{l\{m\}, i}\left(\xi_{i}\right)=-\log \left(1-\xi_{l\{m\}, i}\right)-\frac{\xi_{l\{m\}, i}}{1-\xi_{l\{m\}, i}} \log \xi_{l\{m\}, i}
$$

is the entropy associated to each sub-density matrix in the product shown in Eq. 228 and $\xi_{l\{m\}, i}$ is the parameter that generates the eigenvalues of these densities matrices. Note that each eigenvalue $\xi=\xi_{l\{m\}, i}$ entails a set of probabilities of the form

$$
p_{n}=(1-\xi) \xi^{n} \quad n=0,1,2,3, \ldots
$$

defined by $\xi_{i}=\beta_{i}^{\prime} /\left(1+\left(1-\beta_{i}^{\prime 2}\right)^{1 / 2}\right)$ for each $l\{m\}$ set.

To compute the total entropy, we have to sum over all possible values of $\{m\}$ and $l$.

$$
S=\sum_{l\{m\}} S_{l\{m\}} .
$$

We realize from Eq. 20 that $H_{l\{m\}}$ only depends on $l$, so the entropy associated to its ground state will also be $\{m\}$ independent, and therefore

$$
S=\sum_{l=0}^{\infty} \nu(l, D) S_{l},
$$

being $\nu(l, D)$ the degeneracy of the total angular momentum operator $\hat{L}^{2}$ for a fixed $l$. In three dimensions, for example, $\{m\}=m$ can go from $-l$ to $l$ so that $\nu(l, 3)$ is $2 l+1$. The 
same computation in $D$ dimensions requires the computaiton of the degeneracy of $S O(D)$ representations

$$
\nu(l, D)=\left(\begin{array}{c}
l+D-1 \\
l
\end{array}\right)-\left(\begin{array}{c}
l+D-3 \\
l-2
\end{array}\right) .
$$

Given the explicit knowledge of all the eigenvalues of the reduced density matrix, we can also obtain a formula for the single copy entanglement Eq. 2. The largest eigenvalue of density matrix for two coupled harmonic oscillators is $(1-$ $\xi)$. This largest eigenvalue of the density matrix $\rho_{\text {out }}$ will be the product of the largest eigenvalues of the density matrices which compound $\rho_{\text {out }}$,

$$
\begin{aligned}
\rho_{\text {out }}^{(1)} & =\prod_{l\{m\}} \prod_{i=1}^{N-n}\left(1-\xi_{l\{m\}, i}\right) \\
& =\prod_{l=0}^{\infty}\left(\prod_{i=1}^{N-n}\left(1-\xi_{l\{m\}, i}\right)\right)^{\nu(l, D)} .
\end{aligned}
$$

The single copy entanglement finally reads

$$
E_{1}\left(\rho_{L}\right)=-\sum_{l=0}^{\infty} \nu(l, D)\left(\sum_{i=1}^{N-n} \log \left(1-\xi_{i}\right)\right)
$$

\section{Perturbative computation for large angular momenta}

Note that our expressions for the entropy and the single copy entanglement depend on a final sum that ranges over all the values of angular momentum $l$. This sum may not be convergent as the radial discretization we have implemented is not a complete regularization of the field theory. To be precise, the asymptotic dependence on $l$ should be under control in order to correctly assess the convergence of the series.

Let us note that, for $l \gg N$, the non diagonal elements of $K$ Eq.23 are much smaller than the diagonal ones. These suggests the possibility of setting up a perturbative computation.

We split up the $K$ matrix in a diagonal $K_{0}$ and non diagonal $\lambda \eta$ matrices, where parameter $\lambda$ is just introduced to account for the order in a perturbative expansion of the non-diagonal piece,

$$
K=K_{0}+\lambda \eta .
$$

This expansion is somewhat tedious and non illuminating. Technical details are presented in Appendix A. The main observation is that the first contribution $i=1$ out of every set of $\xi_{l,\{m\}, i}$ elements is relevant and it can further be expanded as a series in $l^{-1}$,

$$
\xi \equiv \xi_{l,\{m\}, 1}=\frac{1}{l^{4}} \sum_{k=0}^{5} \frac{\xi_{k}}{l^{k}}+O\left(l^{-10}\right)
$$

We can then get the entropy $S_{l\{m\}}$.

$$
\begin{aligned}
S_{l\{m\}} & \simeq S_{l\{m\}, 1}=\sum_{k=1}^{\infty}\left(\frac{1}{k}-\log (\xi)\right) \xi^{k} \\
& =\frac{1}{l^{4}} \sum_{k=0}^{5} \frac{s_{k}+t_{k} \log l}{l^{k}}+O\left(l^{-10}\right),
\end{aligned}
$$

where the coefficients $s_{k}$ and $t_{k}$ are defined in Appendix A. A similar result for the single copy entanglement reads,

$$
\begin{aligned}
E_{1} & \simeq \sum_{l=0}^{\infty}-\nu(l, D) \log (1-\xi)+O\left(l^{-10}\right) \\
& =\sum_{l=0}^{\infty} \nu(l, D) \sum_{k=1}^{\infty} \frac{\xi^{k}}{k} \simeq \sum_{l=0}^{\infty} \nu \sum_{j=1}^{5} \frac{\kappa_{j}}{l^{4+j}}+O\left(l^{-10}\right),
\end{aligned}
$$

where $\kappa_{j}$ are the coefficients of the expansion given also in Appendix A. Finally, using Eq. 34 and defining $\tau_{k} \equiv$ $\sum_{j=0}^{k} \nu_{j} t_{k-j}$ and $\sigma_{k} \equiv \sum_{j=0}^{k} \nu_{j} s_{k-j}$ where $\nu_{j}$ are the coefficients of the degeneracy expansion, we determine the contribution to the total entropy, for $l=l_{0} \ldots \infty$, where $l_{0}$ is big enough such that the approximations are valid,

$$
\begin{aligned}
\Delta S & \simeq \sum_{j}^{5} \sigma_{j}\left(\zeta(6-D+j)-\sum_{l=1}^{l_{0}} \frac{1}{l^{6-D+j}}\right) \\
& -\sum_{j}^{5} \tau_{j}\left(\zeta^{\prime}(6-D+j)+\sum_{l=1}^{l_{0}} \frac{\log l}{l^{6-D+j}}\right)
\end{aligned}
$$

where $\zeta(n)$ is the Riemann Zeta function and $\zeta^{\prime}(n)$ its derivative. Defining $\Lambda_{k} \equiv \sum_{j=0}^{k} \nu_{j} \kappa_{k-j}$, the single copy entanglement becomes,

$$
\Delta E_{1} \simeq \sum_{j=0}^{5} \Lambda_{j}\left(\zeta(6-D+j)-\sum_{l=1}^{l_{0}} \frac{1}{l^{6-D+j}}\right)
$$

The above results show that the sum over angular momenta $l$ converges for $D<5$. A radial discretization of a scalar field theory produces finite results for $D<5$ and needs further regularization in orthogonal (angular) directions to the radius in higher dimensions. We will come back to this question later.

\section{Area law scaling}

The analysis of the scaling law obeyed by the geometric entropy proceeds as follows. The analytical treatment of the chain of oscillators lead to the final sum over angular momenta in Eq. 33. The computation of this sum requires polynomial, rather than exponential, effort as the size of the system increases. This justifies why large systems are accessible within this approach. The eigenmodes $\xi_{l\{m\}, i}$ are obtained by diagonalization of matrices of order less than $N$. Finally the tail of 
the sum over angular momenta is computed using the asymptotic expressions given in Eq.41].

We have computed the geometrical entropy and the singlecopy entanglement for different dimensionalities of the system. Within the range $1<D<5$ we do observe the expected area law scaling

$$
S=k_{S}(\mu, D, a, N)\left(\frac{R}{a}\right)^{D-1},
$$

as well as a similar scaling for the single copy entanglement

$$
E_{1}=k_{E}(\mu, D, a, N)\left(\frac{R}{a}\right)^{D-1},
$$

where in all our considerations the lattice spacing can be taken $a=1$. Fig 1 shows this perfect scaling for both measures of entanglement.

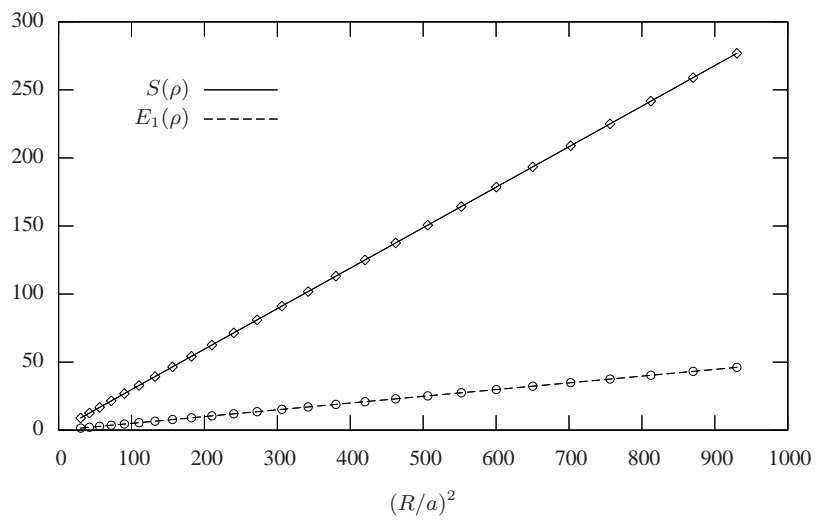

Figure 1: The entropy $S$ and the single copy entanglement $E_{1}$ resulting from tracing the ground state of a massless scalar field in three dimensions, over the degrees of freedom inside a sphere of radius $R$.

The explicit pre-factor in the area law is regularization dependent but can be computed and compared with previous analysis. Fig 2 shows the result obtained for this pre-factor in the area law for the case of $D=3$ and $\mu=0$ as the size of the system increases.

Good stability is already reached for $N=600$, where we recover the result of [2] and complete it with the single copy entanglement

$$
\begin{aligned}
& k_{S}(\mu=0, D=3, N \rightarrow \infty)=0.295(1), \\
& k_{E}(\mu=0, D=3, N \rightarrow \infty)=0.0488(1) .
\end{aligned}
$$

Let us note that the ratio of the area law pre-factors for the entropy and the single copy entanglement is close to 6 . This value is much larger than the factor of 2 computed to be the exact ratio in one-dimensional critical systems [9]. We thus conclude that the amount of entanglement that can be extracted from a single copy of a system as compared to the asymptotic value for infinite copies does decrease with the dimensionality.

We can analyze in more detail the dependence of our two measures of entanglement as a function of the dimensionality

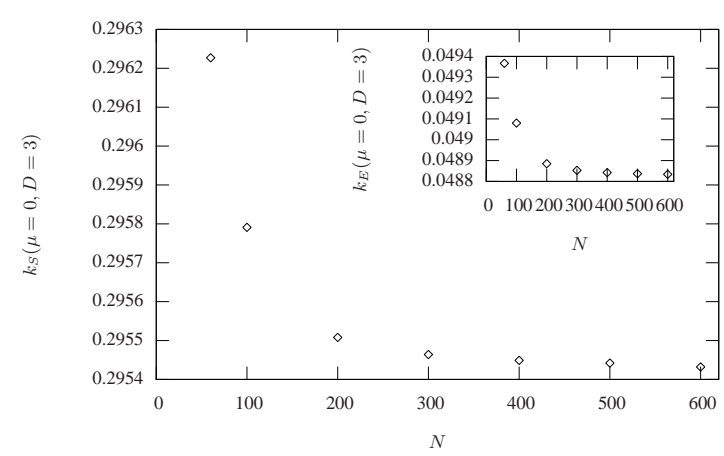

Figure 2: Coefficient for entropy the area law in $D=3$ as a function of size of the system. Good stability is reached for $N=600$. In the inset, the corresponding coefficient for the single copy area law is plotted.

of the system. This is shown in Fig 3 for an $N=60$ and $5 \leq n \leq 30$ as a function of $R^{D-1}$ and we verify that the area law is observed for any value of the dimension $D$.

The robustness of area scaling law for arbitrary mass $\mu$ is also readily checked (see Fig 5. The appearance of a mass term in the Hamiltonian produces exponential decays of correlators but does not affect the short-distance entanglement which is ultimately responsible for the area law. This supports the idea that geometric entropy comes from the local neighborhood of the surface separating the region which is integrated out. The exponential decay of massive modes is immaterial and their contribution to the entanglement entropy is as important as the one coming from massless modes.

Let us concentrate briefly in the dependence of $k_{S}$ and $k_{E}$ on the dimension $D$. Those coefficients present divergences at $D=1$ and $D=5$ (see Fig. 3). The first one is due to the fact that in one dimension the strict power area law breaks down, since the limiting case carries a logarithmic dependence. For $D \geq 5$, as we have shown before, the sum over partial waves does not converge. This is due to the fact that we have regularized the Hamiltonian using a radial lattice. This regularization is insufficient to handle higher dimensional modes due to the increase of degrees of freedom per radial shell. To avoid this problem, a more elaborated regularization of the initial $D$-dimensional Hamiltonian is required. Such a regularization will likely have to break the rotational symmetry and will make the computations rather involved.

We observe in Fig 4 that the entropy to single-copy entanglement ratio verifies the expected limit 2, for $D$ tending to 1.

\section{E. Vacuum reordering}

Area law implies that entropy grows with the size of the system, that is, the eigenvalues of the density matrix, properly sorted from the largest to the smallest, decay in a slower way for larger systems. It has been numerically shown in Ref.[48] that this order relation between systems of different length 


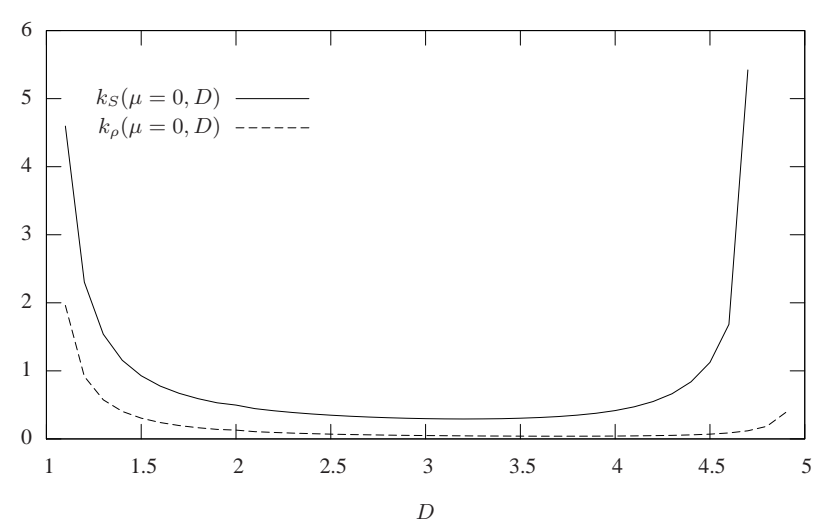

Figure 3: Dependence of the geometric entropy and single copy entanglement slopes, $k_{S}$ and $k_{E}$, on the dimension $D$ for a massless scalar field. Note the divergence at $D=5$ due to the insufficient radial regularization of the original field theory.

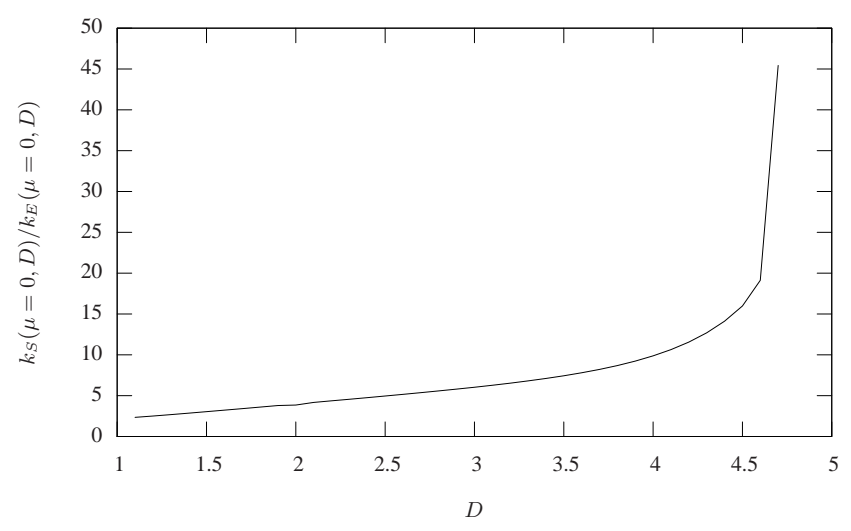

Figure 4: Evolution of entropy to single-copy entanglement ratio $S / E_{1}$ as a function of the dimension $D$. The line starts at a value of 2, as demonstrated analytically in [9] and grows monotonically. The higher the dimension is, the less entanglement is carried by a single copy of the system as compared to many copies.

verifies the strong condition of majorization, a fact proven analytically for conformal field theories in Ref.[49]. As the size of the system increases from $L$ to $L^{\prime}>L$, it is verified that $\rho_{L^{\prime}} \prec \rho_{L}$, where $\rho_{L}$ and $\rho_{L^{\prime}}$ are the set of eigenvalues for the corresponding reduced density matrices.

Majorization relations characterize strong ordering. Every eigenvalue changes in a way that is consistent with a set of majorization constraints. We shall refer to this fact as vacuum reordering.

We show that the same underlying reordering of the vacuum is present in any number of dimensions. Unfortunately, a similar analytical treatment to the $D=1$ case is out of reach because the conformal group in $D>1$ is spanned by a finite number of generators. As a consequence, there is no full control on the partition function of conformal field theory in $D>1$ dimensions, which could be used to generalize the one-dimensional theorem.

Vacuum reordering can be treated within our semi numer- ical approach. From Eq. 28, we see that the reduced density matrix of the exterior of a ball of radius $R$, can be expressed as a tensor product of simpler density matrices,

$$
\rho_{\text {out }}(R)=\prod_{l\{m\}} \rho_{l\{m\}}(R)=\prod_{l\{m\}}\left(\prod_{i=1}^{N-n} \rho_{l\{m\}, i}(R)\right),
$$

where $\rho_{l\{m\}}$ is what we call $\rho_{\text {out }}$ in Sec. (IIIB and $\rho_{l\{m\}, i}$ are defined in the same section. A similar composition applies for another size $R^{\prime}$,

$$
\rho_{\text {out }}\left(R^{\prime}\right)=\prod_{l\{m\}} \rho_{l\{m\}}\left(R^{\prime}\right)=\prod_{l\{m\}}\left(\prod_{i=1}^{N-n^{\prime}} \rho_{l\{m\}, i}\left(R^{\prime}\right)\right) .
$$

It is shown as a lemma in Ref.[48] that, if majorization relations are satisfied by each $\rho_{l\{m\}}(R)$ and $\rho_{l\{m\}}\left(R^{\prime}\right)$, they will be also satisfied by $\rho(R)$ and $\rho\left(R^{\prime}\right)$. Note, though, that it is not possible to follow the same argument for $\rho_{l\{m\}, i}(R)$ and $\rho_{l\{m\}, i}\left(R^{\prime}\right)$ since $n \neq n^{\prime}$. To make dimensions agree, we need to complete with identity operators the smallest set. We then find that some majorization relations for the subparts are obeyed in one sense, and the rest in the opposite one. Thus, we construct the density matrices $\rho_{l\{m\}}(R)$ and $\rho_{l\{m\}}\left(R^{\prime}\right)$ doing the tensorial product of their components which are generated using Eq.31. Once we have their eigenvalues we are ready to check that if $R<R^{\prime}$, then

$$
\rho_{\text {out }}\left(R^{\prime}\right) \prec \rho_{\text {out }}(R),
$$

which means by definition

$$
\sum_{i=1}^{k} p_{i}^{\prime} \leq \sum_{i=1}^{k} p_{i} \forall k=1, \ldots, \infty
$$

where $p_{i}$ and $p_{i}^{\prime}$ are the eigenvalues of $\rho_{\text {out }}(R)$ and $\rho_{\text {out }}\left(R^{\prime}\right)$ respectively. For the $l \sim N$ case, we have done a numerical computation with $N=60$ and truncating the vector of eigenvalues at the 50th element. Several dimensions $D$ and traced sizes $n$ have been studied, and all majorization relations are satisfied in all of them, as expected. When $l \gg N$, we can use the analytical results of the Appendix A to check the same result.

\section{ENTANGLEMENT LOSS ALONG RG TRAJECTORIES}

We shall now exploit the control achieved on the eigenvalues of the reduced density matrix in $D$ dimensions to study how entanglement evolves along renormalization group transformations. This was studied for the quantum Ising model in Ref. [48]. We shall now add equivalent results for the set of harmonic oscillators in $D$ dimensions. Results will turn out to be qualitatively similar, reinforcing the concept of entanglement loss along RG flows.

The renormalization of a bosonic field is particularly simple since the Hamiltonian only carries one coupling, namely 
the mass term. After a block transformation, the rescaling of fields is used to make the kinetic term to be normalized to $\frac{1}{2}$. The RG flow of the massive scalar field reduces to an effective change of the mass. That is, the study of the long distance behavior of a correlator is viewed as taking a larger mass for the field, modulo a scaling factor. This implies the existence of two fixed points which are $\mu=0$ (ultraviolet, UV) and $\mu=\infty$ (infrared, IR). Since no other fixed point is possible, the RG flow must be monotonic in $\mu$.

Entanglement loss comes along this flow. First, we study this change from a global perspective. We observe the obvious global loss of entanglement. For $\mu=0$, geometric entropy grows with a slope $k_{S}(D, \mu=0)$ for the massless field and it is zero for the $\mu=\infty$ case. Thus,

$$
S_{U V} \geq S_{I R} \quad \forall R
$$

This result is related to the c-theorem as discussed in Refs. [50, 51, 52, 53, 54], which states global irreversibility in the RG trajectory which interpolates between UV and IR fixed points.

On top of this global loss of entanglement, the geometric entropy obeys a monotonic decrease along the RG flow. This behavior is illustrated for $D=3$ in Fig 5 the entropy for different masses where it is seen that

$$
\mu^{\prime}>\mu \Longrightarrow k_{S}\left(\mu^{\prime}\right)<k_{S}(\mu)
$$

Thus, the system is more ordered as the mass increases.

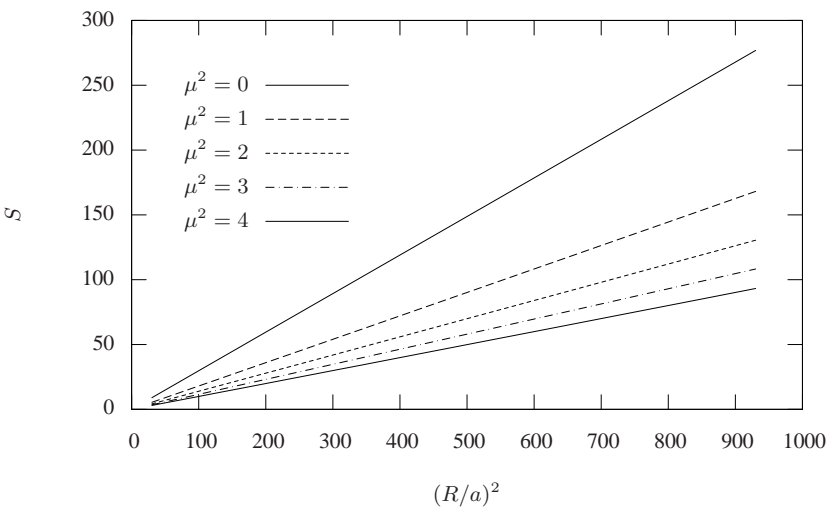

Figure 5: Geometric entropy $S$ for a sphere of radius $R$ in $D=3$ as a function of the mass $\mu$. Note that larger masses produce a smaller coefficient in the scaling are law.

It is natural to pose the question if this order relation verifies also stricter majorization relations, that is, vacuum reordering. Specifically, we analyze whether $\rho\left(\mu^{\prime}\right)$ and $\rho(\mu)$, the density matrices corresponding to the free bosonic model with masses $\mu^{\prime}>\mu$ respectively, obey $\vec{p}\left(\mu^{\prime}\right)$ and $\vec{p}(\mu)$.

$$
\vec{p}(\mu) \prec \vec{p}\left(\mu^{\prime}\right) .
$$

Using similar arguments as in the previous section, we only need to check that each $\rho_{l\{m\}, i}(\mu)$ majorizes $\rho_{l\{m\}, i}\left(\mu^{\prime}\right)$.

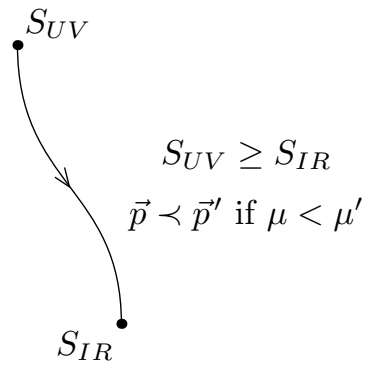

Figure 6: Entanglement loss along the RG trajectories seen in the space spanned by the eigenvalues of the reduced density matrix.

Considering Eq.31, that means,

$$
\sum_{i=1}^{k}(1-\xi) \xi^{i} \leq \sum_{i=1}^{k}\left(1-\xi^{\prime}\right) \xi^{i} \quad \forall k=1, \ldots, \infty,
$$

and therefore,

$$
\left(1-\xi^{k+1}\right) \leq\left(1-\xi^{k+1}\right) \quad \forall k=1, \ldots, \infty .
$$

This happens if and only if $\xi^{\prime} \leq \xi$. As in the previous section, we have verified this fact numerically in the $l \sim N$ regime, and analytically using the perturbation calculus done in Appendix A.

It should be noted that monotonic loss of entanglement is mandatory in such a simple model with a single parameter $(\mu)$ controlling the flow. It is far from obvious that such entropy loss is rooted in a such a subtle reordering of the vacuum as the one dictated by majorization.

\section{CONCLUSIONS}

Area law scaling for the geometric entropy is present in harmonic networks of arbitrary dimensions. This follows from a computation that makes use of and analytical approach capable of making an analytical extension of the computation to arbitrary $D$, followed by a final numerical resummation of angular momenta, whose tail is controlled analytically.

A similar scaling law is observed for the single-copy entanglement. This result suggests that entanglement, whatever measure we use, scales with an area law due to the fact that entanglement is concentrated on the surface of the region which is traced out. The ratio of single-copy entanglement to geometric entropy tends to zero as the dimension of the network increases.

It is natural to interprete a change in the size of the subsystem which is traced out as well as any modification of the parameters in the Hamiltonian as a probe on the vacuum. Our explicit computations unveil ubiquous vacuum reordering governed by majorization relations of the vacuum state reduced density matrix eigenvalues. Geometric entropy scaling is just one manifestation of this set of order relations.

The fact that finite PEPS support an area law scaling makes them a natural tool to investigate regularized quantum field theories. 


\section{Appendix A: PERTURBATION THEORY}

We need to perform a perturbative computation for large momenta in order to determine the contribution to the total entropy and single copy entanglement of all angular momentum modes.

We organize our computation in three parts. In the first part, we carry out perturbation theory with matrices, following the same steps as explained in sec. (IIIB) when considering the aproximation $l \gg N$. This will produce an analitical expression for the $\xi$ 's parameters. The second part of the computation consists in Taylor expanding the above results for $\xi$ in a series in $l^{-1}$. Finally, we will get the entropy and single copy entanglement contributions, expanding the entropy and single copy modes in terms of $l^{-1}$ powers, and summing over $l$. In this sum $l$ take values from $l_{0}$ until infinity, where $l_{0}$ must be suffitiently large, such that all aproximations done previously are right.

\section{Computation of the $\xi$ parameter}

Let us recall that, for $l \gg N$, the non diagonal elements of $K$ in Eq. 23 are much smaller than the diagonal ones. That gives us the possibility of setting up a perturbative computation.

We split up the $K$ matrix in a diagonal $K_{0}$ and non diagonal $\lambda \eta$, matrices where $\lambda$ is just introduced to account for the order in a perturbative expansion of the non-diagonal piece,

$$
K=K_{0}+\lambda \eta \text {. }
$$

We will follow the steps described in Sec MB We expand $\Omega \equiv \sqrt{K}$ in its different contributions to order $\lambda$,

$$
\Omega=\Omega_{0}+\lambda \epsilon+\lambda^{2} \tilde{\epsilon}+\lambda^{3} \hat{\epsilon}+O\left(\lambda^{4}\right) .
$$

To get each term we impose the condition $\Omega^{2}=K$,

$$
\begin{aligned}
\left(\Omega_{0}\right)_{i j} & =\Omega_{i} \delta_{i j} \\
(\epsilon)_{i j} & =\epsilon_{i} \delta_{i+1, j}+\epsilon_{j} \delta_{j+1, i} \\
(\tilde{\epsilon})_{i j} & =\frac{\epsilon_{i}^{2}+\epsilon_{i-1}^{2}}{\Omega_{i}+\Omega_{j}} \delta_{i j}+\frac{\epsilon_{i} \epsilon_{j-1}}{\Omega_{i}+\Omega_{j}} \delta_{i+2, j} \\
& +\frac{\epsilon_{j} \epsilon_{i-1}}{\Omega_{i}+\Omega_{j}} \delta_{i, j+2} \\
(\hat{\epsilon})_{i j} & =\frac{(\epsilon \tilde{\epsilon}+\tilde{\epsilon} \epsilon)_{i j}}{\Omega_{i}+\Omega_{j}}
\end{aligned}
$$

where $\Omega_{j}$ and $\epsilon_{j}$ are defined since

$$
\begin{aligned}
\Omega_{j} & \equiv \sqrt{\frac{l(l+D-2)}{j^{2}}+\omega_{j}} \\
\omega_{j} & \equiv\left(1+\frac{1}{2 j}\right)^{D-1}+\left(1-\frac{1}{2 j}\right)^{D-1}+\mu^{2}, \\
\epsilon_{j} & \equiv-\frac{j+\frac{1}{2}}{\sqrt{j(j+1)}} \frac{1}{\Omega_{j}+\Omega_{j+1}} .
\end{aligned}
$$

We structure $\Omega$ in three matrices $A, B$ and $C$,

$$
\begin{aligned}
A & \equiv \Omega(1 \div n, 1 \div n)=A_{0}+\lambda A_{1}+\lambda^{2} A_{2}+O\left(\lambda^{3}\right) \\
B & \equiv \Omega(1 \div n, n+1 \div N)= \\
& =\lambda B_{0}+\lambda^{2} B_{1}+\lambda^{3} B_{2}+O\left(\lambda^{4}\right) \\
C & \equiv \Omega(n+1 \div N, n+1 \div N)= \\
& =C_{0}+\lambda C_{1}+\lambda^{2} C_{2}+O\left(\lambda^{3}\right) .
\end{aligned}
$$

From these matrices, we define $\beta$ and $\gamma$ which we write in series of $\lambda$

$$
\begin{aligned}
& \beta \equiv \frac{1}{2} B^{T} A^{-1} B=\lambda^{2} \beta_{0}+\lambda^{3} \beta_{1}+\lambda^{4} \beta_{2}+O\left(\lambda^{4}\right) \\
& \gamma \equiv C-\beta=\Omega_{0}+\lambda \epsilon+O\left(\lambda^{2}\right),
\end{aligned}
$$

where,

$$
\begin{aligned}
\beta_{0} & =\frac{1}{2} B_{0}^{T} A_{0}^{-1} B_{0}=\frac{\epsilon_{n}^{2}}{2 \Omega_{n}} \delta_{i, 1} \delta_{j, 1} \\
\beta_{1} & =\frac{1}{2}\left(B_{1}^{T} A_{0}^{-1} B_{0}+B_{0}^{T} A_{0}^{-1} B_{1}+B_{0}^{T} A_{1}^{-1} B_{0}\right) \\
& =-\frac{\epsilon_{n}^{2}}{2 \Omega_{n}} \frac{\epsilon_{n+1}}{\Omega_{n}+\Omega_{n+2}}\left(\delta_{i, 2} \delta_{j, 1}+\delta_{i, 1} \delta_{j, 2}\right) \\
\beta_{2} & =\frac{1}{2}\left(B_{2}^{T} A_{0}^{-1} B_{0}+B_{0}^{T} A_{0}^{-1} B_{2}+B_{0}^{T} A_{2}^{-1} B_{0}\right. \\
& \left.+B_{1}^{T} A_{0}^{-1} B_{1}+B_{1}^{T} A_{1}^{-1} B_{0}+B_{0}^{T} A_{1}^{-1} B_{1}\right) .
\end{aligned}
$$

We shall see later, that at $2 \mathrm{n}$ order perturbation in $\lambda$, only $\left(\beta_{2}\right)_{11}$ and $\left(\beta_{2}\right)_{22}$ of $\beta_{2}$ are necessary. Then,

$$
\begin{aligned}
\left(\beta_{2}\right)_{11} & =\frac{\epsilon_{n}^{2}}{2 \Omega_{n}}\left\{\frac{\epsilon_{n-1}^{2}}{\Omega_{n} \Omega_{n-1}}+\frac{\epsilon_{n-1}^{2}+\epsilon_{n}^{2}}{2 \Omega_{n}^{2}}\right. \\
& +\frac{\Omega_{n}}{\Omega_{n-1}} \frac{\epsilon_{n-1}^{2}}{\left(\Omega_{n+1}+\Omega_{n-1}\right)^{2}}+\frac{2 \epsilon_{n-1}^{2}}{\Omega_{n-1}\left(\Omega_{n+1}+\Omega_{n-1}\right)} \\
& +\frac{2}{\Omega_{n}\left(\Omega_{n}+\Omega_{n+1}\right)}\left(\frac{\epsilon_{n+1}^{2}}{\Omega_{n}+\Omega_{n+2}} \frac{\epsilon_{n+1}^{2}+\epsilon_{n}^{2}}{2 \Omega_{n+1}}\right. \\
& \left.\left.+\frac{\epsilon_{n}^{2}+\epsilon_{n-1}^{2}}{2 \Omega_{n}}+\frac{\epsilon_{n-1}^{2}}{\Omega_{n+1}+\Omega_{n-1}}\right)\right\} \\
\left(\beta_{2}\right)_{22} & =\frac{\epsilon_{n}^{2}}{2 \Omega_{n}} \frac{\epsilon_{n+1}^{2}}{\left(\Omega_{n}+\Omega_{n+2}\right)^{2}} .
\end{aligned}
$$

Let us diagonalize $\gamma$,

$$
\gamma_{D}=V \gamma V^{T}
$$

where $V$ is an orthogonal matrix $\left(V V^{T}=\mathbb{1}\right)$. Therefore, the eigenvalues are

$$
\begin{aligned}
\operatorname{det}(\gamma-w \mathbb{1}) & =\prod_{i=1}^{N-n}\left(\Omega_{n+i}-w\right)+O\left(\lambda^{2}\right)=0 \\
\Rightarrow w_{i} & =\Omega_{n+i}+O\left(\lambda^{2}\right)
\end{aligned}
$$

and

$$
\left(\gamma_{D}\right)_{i j}=\left(\Omega_{n+i}+O\left(\lambda^{2}\right)\right) \delta_{i j}
$$


If we impose ager $V=V_{0}+\lambda V_{1}+\lambda^{2} V_{2}+O\left(\lambda^{3}\right)$, we obtain,

$$
\begin{aligned}
V_{0} & =\mathbb{1} \\
\left(V_{1}\right)_{i j} & =\frac{\epsilon_{n+i}}{\Omega_{n+i}-\Omega_{n+j}} \delta_{i+1, j}+\frac{\epsilon_{n+j}}{\Omega_{n+j}-\Omega_{n+i}} \delta_{i, j+1} \\
\left(V_{2}\right)_{11} & =\frac{1}{2}\left(\frac{\epsilon_{n+1}}{\Omega_{n+1}-\Omega_{n+2}}\right)^{2} .
\end{aligned}
$$

Once we have $V$ and $\gamma_{D}$ we are able to compute $\beta^{\prime}=\lambda^{2}\left(\beta_{0}^{\prime}+\right.$ $\left.\lambda \beta_{1}^{\prime}+\lambda^{2} \beta_{2}^{\prime}+O\left(\lambda^{3}\right)\right)$, which is defined by,

$$
\beta^{\prime} \equiv \gamma_{D}^{-\frac{1}{2}} V \beta V^{T} \gamma_{D}^{-\frac{1}{2}}
$$

Thus,

$$
\begin{aligned}
\beta_{0}^{\prime} & =\left(\gamma_{D}^{-\frac{1}{2}}\right)_{0} \beta_{0}\left(\gamma_{D}^{-\frac{1}{2}}\right)_{0} \\
\beta_{1}^{\prime} & =\left(\gamma_{D}^{-\frac{1}{2}}\right)_{0}\left[\beta_{1}+V_{0} \beta_{1}+\beta_{1} V_{0}^{T}+V_{1} \beta_{0}+\beta_{0} V_{1}^{T}\right]\left(\gamma_{D}^{-\frac{1}{2}}\right)_{0} \\
\beta_{2}^{\prime} & =\left(\gamma_{D}^{-\frac{1}{2}}\right)_{2} \beta_{0}\left(\gamma_{D}^{-\frac{1}{2}}\right)_{0}+\left(\gamma_{D}^{-\frac{1}{2}}\right)_{0} \beta_{0}\left(\gamma_{D}^{-\frac{1}{2}}\right)_{2} \\
& +\left(\gamma_{D}^{-\frac{1}{2}}\right)_{0}\left[\beta_{2}+V_{1} \beta_{1}+\beta_{1} V_{1}^{T}+V_{2} \beta_{0}+\beta_{0} V_{2}^{T}\right. \\
& \left.+V_{1} \beta_{0} V_{1}^{T}\right]\left(\gamma_{D}^{-\frac{1}{2}}\right)_{0}
\end{aligned}
$$

and therefore,

$$
\begin{aligned}
\left(\beta_{0}^{\prime}\right)_{i j} & =\frac{\epsilon_{n}^{2}}{2 \Omega_{n+1} \Omega_{n}} \delta_{i, 1} \delta_{j, 1} \\
\left(\beta_{1}^{\prime}\right)_{i j} & =\frac{\epsilon_{n}^{2}}{2 \Omega_{n+1} \Omega_{n}} \sqrt{\frac{\Omega_{n+1}}{\Omega_{n+2}} \epsilon_{n+1}} \\
& \times\left(\frac{1}{\Omega_{n+1}-\Omega_{n+2}}+\frac{1}{\Omega_{n}+\Omega_{n+2}}\right)\left(\delta_{i, 1} \delta_{j, 2}+\delta_{i, 2} \delta_{j, 1}\right) \\
\left(\beta_{2}^{\prime}\right)_{11} & =\frac{\left(\beta_{2}\right)_{11}}{\Omega_{n+1}}-\frac{\epsilon_{n}^{2}}{2 \Omega_{n} \Omega_{n+1}} \frac{\epsilon_{n+1}^{2}}{\left(\Omega_{n}-\Omega_{n-1}\right)^{2}} \\
& -\frac{\epsilon_{n}^{2}}{2 \Omega_{n} \Omega_{n+1}}\left(\frac{2 \epsilon_{n+1}^{2}}{\left(\Omega_{n+2}+\Omega_{n}\right)\left(\Omega_{n+1}-\Omega_{n+2}\right)}\right. \\
& \left.-\frac{1}{2 \Omega_{n+1}^{2}}\left(\epsilon_{n+1}^{2} \frac{\Omega_{n+1}+\Omega_{n+2}}{\Omega_{n+1}-\Omega_{n+2}}-\epsilon_{n}^{2} \frac{\Omega_{n}+\Omega_{n+1}}{\Omega_{n}}\right)\right) \\
\left(\beta_{2}^{\prime}\right)_{22} & =\frac{\epsilon_{n}^{2} \epsilon_{n+1}^{2}}{2 \Omega_{n+2} \Omega_{n}}\left(\frac{1}{\Omega_{n+1}-\Omega_{n+2}}+\frac{1}{\Omega_{n}+\Omega_{n+2}}\right)^{2} .
\end{aligned}
$$

It will be useful to write $\beta^{\prime}$ in its matrix form,

$$
\beta^{\prime}=\lambda^{2}\left(\begin{array}{cccc}
a_{n}+\lambda^{2} c_{n} & \lambda d_{n} & 0 & \ldots \\
\lambda d_{n} & \lambda^{2} e_{n} & 0 & \ldots \\
0 & 0 & 0 & \ldots \\
\vdots & \vdots & \vdots & \ddots
\end{array}\right)+O\left(\lambda^{5}\right),
$$

where,

$$
\begin{aligned}
& a_{n} \equiv \frac{\epsilon_{n}^{2}}{2 \Omega_{n+1} \Omega_{n}} \\
& d_{n} \equiv a_{n} \sqrt{\frac{\Omega_{n+1}}{\Omega_{n+2}}} \epsilon_{n+1}\left(\frac{1}{\Omega_{n+1}-\Omega_{n+2}}+\frac{1}{\Omega_{n}+\Omega_{n+2}}\right)
\end{aligned}
$$

and $c_{n}$ and $e_{n}$ are respectively $\left(\beta_{2}^{\prime}\right)_{11}$ and $\left(\beta_{2}^{\prime}\right)_{22}$. We can observe now, that if we had not found the second order contribution of $\left(\beta^{\prime}\right)_{11}$ and $\left(\beta^{\prime}\right)_{22}$, we would not have been able to compute the eigenvalues of $\beta^{\prime}$ to this order.

Diagonalizing $\beta^{\prime}$, we find the eigenvalues $v_{1}$ and $v_{2}$,

$$
\begin{aligned}
& v_{1}=\lambda^{2}\left(a_{n}+\lambda^{2}\left(c_{n}+\frac{d^{2}}{a_{n}}\right)+O\left(\lambda^{3}\right)\right) \\
& v_{2}=\lambda^{4}\left(e_{n}-\frac{d_{n}^{2}}{a_{n}}\right)+O\left(\lambda^{5}\right)=0+O\left(\lambda^{5}\right),
\end{aligned}
$$

which allows us to compute the $\xi_{i}$ 's parameters,

$$
\xi_{i}=\frac{v_{i}}{1+\sqrt{1-v_{i}^{2}}},
$$

and which read

$$
\begin{aligned}
& \xi_{1}=\frac{\lambda^{2}}{2}\left(a_{n}+\lambda^{2}\left(c_{n}+\frac{d^{2}}{a_{n}}\right)+O\left(\lambda^{3}\right)\right) \\
& \xi_{2}=0+O\left(\lambda^{5}\right) \\
& \xi_{i}=O\left(\lambda^{7}\right) \forall i>2 .
\end{aligned}
$$

\section{Expansion of $\xi$ in terms of $l^{-1}$ powers}

We rename $\xi_{1}$ as $\xi$, and neglect the rest since at this order they are 0 and no contribute neither to the entropy nor to the single copy entanglement. We are interested in expanding $\xi$ in powers of $l^{-1}$. To do this, we have to expand first $\Omega_{j}$ and $\epsilon_{j}$,

$$
\Omega_{n}=l \sum_{i=0}^{9} \frac{\Omega_{n}^{(i)}}{l^{i}}+O\left(l^{-9}\right),
$$

where,

$$
\begin{aligned}
\Omega_{n}^{(0)} & =\frac{1}{n} \\
\Omega_{n}^{(1)} & =\frac{D-2}{2 n} \\
\Omega_{n}^{(2)} & =\frac{n \omega_{n}}{2}+\frac{(D-2)^{2}}{8 n} \\
\Omega_{n}^{(3)} & =\frac{(D-2)^{3}-4(D-2) n^{2} \omega_{n}}{16 n} \\
\Omega_{n}^{(4)} & =-\frac{5(D-2)^{4}-24(D-2)^{2} n^{2} \omega_{n}+16 n^{4} \omega_{n}^{2}}{128 n} \\
\Omega_{n}^{(5)} & =\frac{7(D-2)^{5}}{256 n} \\
& +\frac{-40(D-2)^{2} n^{2} \omega_{n}+48 n^{4} \omega_{n}^{2}}{256 n}
\end{aligned}
$$

No more coefficients have been presented here since they have huge expressions and they don't shed any light on our arguments. Using $\Omega_{n}$ we can obtain the expansion of $\epsilon_{n}$,

$$
\epsilon_{n}=\frac{1}{l} \sum_{i=0}^{6} \frac{\epsilon_{n}^{(i)}}{l^{i}}+O\left(l^{-8}\right),
$$


where,

$$
\begin{aligned}
& \epsilon_{n}^{(0)}=\frac{(\eta)_{n, n+1}}{\Omega_{n}^{(0)}+\Omega_{n+1}^{(0)}} \\
& \epsilon_{n}^{(1)}=-\epsilon_{n}^{(0)} \frac{\Omega_{n}^{(1)}+\Omega_{n+1}^{(1)}}{\Omega_{n}^{(0)}+\Omega_{n+1}^{(0)}} \\
& \epsilon_{n}^{(2)}=\epsilon_{n}^{(0)}\left(\left(\frac{\Omega_{n}^{(1)}+\Omega_{n+1}^{(1)}}{\Omega_{n}^{(0)}+\Omega_{n+1}^{(0)}}\right)^{2}-\frac{\Omega_{n}^{(2)}+\Omega_{n+1}^{(2)}}{\Omega_{n}^{(0)}+\Omega_{n+1}^{(0)}}\right)
\end{aligned}
$$

Once we have $\Omega_{n}$ and $\epsilon_{n}$ in series of $l^{-1}$, we can expand $\xi$,

$$
\xi=\frac{1}{l^{4}} \sum_{i=0}^{6} \frac{\xi_{i}}{l^{i}}+O\left(l^{-10}\right),
$$

with

$$
\begin{aligned}
& \xi_{0}=\frac{\left(\epsilon_{n}^{(0)}\right)^{2}}{4 \Omega_{n}^{(0)}+\Omega_{n+1}^{(0)}} \\
& \xi_{1}=\epsilon_{n}^{(0)} \frac{2 \epsilon_{n}^{(1)} \Omega_{n}^{(0)} \Omega_{n+1}^{(0)}-\epsilon_{n}^{(0)}\left(\Omega_{n}^{(1)} \Omega_{n+1}^{(0)}+\Omega_{n}^{(0)} \Omega_{n+1}^{(1)}\right)}{4\left(\left(\Omega_{n}^{(0)}\right)^{2}+\left(\Omega_{n+1}^{(0)}\right)^{2}\right)}
\end{aligned}
$$

Although $\xi$ depends on the number of oscillators which we trace out, we have omitted the subindex $n$ to simplify the notation.

\section{The entropy}

The contribution to the entropy of a $(l,\{m\})$-mode becomes,

$$
\begin{aligned}
S_{l\{m\}} & =-\log \left(1-\xi_{l}\right)-\frac{\xi_{l}}{1-\xi} \log \xi_{l} \\
& \simeq \sum_{k=1}^{\infty}\left(\frac{1}{k}-\log (\xi)\right) \xi^{k} .
\end{aligned}
$$

If we substitute $\xi$,

$$
S_{l\{m\}}=\frac{1}{l^{4}} \sum_{k=0}^{5} \frac{s_{k}+t_{k} \log l}{l^{k}}+O\left(l^{-10}\right),
$$

where,

$$
\begin{aligned}
s_{0} & =\xi_{0}-\xi_{0} \log \xi_{0} \\
s_{1} & =-\xi_{1} \log \xi_{0} \\
s_{2} & =-\frac{\xi_{1}^{2}}{2 \xi_{0}}-\xi_{2} \log \xi_{0} \\
s_{3} & =\frac{\xi_{1}^{3}-6 \xi_{0} \xi_{1} \xi_{2}}{6 \xi_{0}^{2}}-\xi_{3} \log \xi_{0} \\
\vdots & ,
\end{aligned}
$$

and

$$
\begin{aligned}
t_{i} & =4 \xi_{i} \quad 0<i \leq 3 \\
t_{4} & =4\left(\xi_{0}^{2}+\xi_{4}\right) \\
t_{5} & =4\left(2 \xi_{0} \xi_{1}+\xi_{5}\right) .
\end{aligned}
$$

To determine the contribution to the entropy of all modes with the same $l$, we use the expansion of the degeneration,

$$
\begin{aligned}
\nu(l, D) & =\left(\begin{array}{c}
l+D-1 \\
l
\end{array}\right)-\left(\begin{array}{c}
l+D-3 \\
l-2
\end{array}\right) \\
& =l^{D-2} \sum_{k=0}^{\infty} \frac{\nu_{k}(D)}{l^{k}}
\end{aligned}
$$

which allows us to sum over all the possible values of $\{m\}$,

$$
\sum_{\{m\}} S_{l\{m\}}=\nu(l, D) S_{l\{m\}}=\sum_{i=0}^{5} \frac{\sigma_{i} \log l+\tau_{i}}{l^{6-D+i}}+O\left(l^{D-12}\right)
$$

where $\tau_{k} \equiv \sum_{j=0}^{k} \nu_{j} t_{k-j}$ and $\sigma_{k} \equiv \sum_{j=0}^{k} \nu_{j} s_{k-j}$. Finally, we can compute the contribution to total entropy, for $l$ from $l_{0}$ to $\infty$, where $l_{0}$ is big enough such that these aproximations are justified.

$$
\begin{aligned}
\Delta S & \simeq \sum_{j}^{5} \sigma_{j}\left(\zeta(6-D+j)-\sum_{l=1}^{l_{0}} \frac{1}{l^{6-D+j}}\right) \\
& -\sum_{j}^{5} \tau_{j}\left(\zeta^{\prime}(6-D+j)+\sum_{l=1}^{l_{0}} \frac{\log l}{l^{6-D+j}}\right),
\end{aligned}
$$

being $\zeta(k)$ the Riemann Zeta function, and $\zeta^{\prime}(k)$ its derivative.

\section{The single-copy entanglement}

We can do the same as for the entropy to find the contribution to the single-copy entanglement for large values of $l$. First, we expand the contribution to the total single-copy entanglement of the $(l,\{m\})$ modes,

$$
\left(E_{1}\right)_{l\{m\}} \simeq-\log \left(1-\xi_{l}\right)=\sum_{i=0}^{5} \frac{\kappa_{i}}{l^{4+i}}+O\left(l^{-10}\right),
$$


where,

$$
\begin{aligned}
\kappa_{i} & =\xi_{i} \quad 0<i \leq 3 \\
\kappa_{4} & =\left(\frac{\xi_{0}^{2}}{2}+\xi_{4}\right) \\
\kappa_{5} & =\left(\xi_{0} \xi_{1}+\xi_{5}\right) .
\end{aligned}
$$

Next, we sum for all possible values of $\{m\}$, using Eq. A30,

$$
\left(E_{1}\right)_{l}=\nu(l, D)\left(E_{1}\right)_{l\{m\}}=\sum_{i=0}^{5} \frac{\Lambda_{i}}{l^{D-6+i}},
$$

where $\Lambda_{k} \equiv \sum_{j=0}^{k} \nu_{j} \kappa_{k-j}$. Proceeding as before, we finally get

$$
E_{1} \simeq \sum_{j=0}^{5} \Lambda_{j}\left(\zeta(6-D+j)-\sum_{l=1}^{l_{0}} \frac{1}{l^{6-D+j}}\right) .
$$

Acknowledgements.- We would like to thank E. Rico who collaborated in the early discussion on majorization relations. We would also like to acknowledge fruitful discussions with M. Asorey, S. Iblisdir, R. Orús and J.M. Escartín. Finally we are grateful to J. Eisert and M. Cramers. for a starting discussion on the ratio of $E_{1} / S$ in arbitrary dimensions. This work has been supported by MEC (Spain), QAP (EU), Grup consolidat (Generalitat de Catalunya) and Universitat de Barcelona.
[1] W. K. Wootters, Phys. Rev. Lett. 80, 2245 (1998); quantph/9709029.

[2] M. Srednicki, Phys. Rev. Lett. 71666 (1993); hep-th/9303048.

[3] C. G. Callan and F. Wilczek, Phys. Lett.B 333 (1994) 55; hepth/9401072.

C. Holzhey, F. Larsen and F. Wilczek, Nucl. Phys. B424, 443 (1994); hep-th/9403108.

[4] T. M. Fiola, J. Preskill, A. Strominger and S. P. Trivedi, Phys. Rev. D 50 (1994) 3987; hep-th/9403137.

[5] G. Vidal, J. I. Latorre, E. Rico and A. Kitaev, Phys. Rev. Lett. 90, 227902 (2003); quant-ph/0211074.

J. I. Latorre, E. Rico and G. Vidal, Quant. Inf. and Comp. 4 48, (2004); quant-ph/0304098.

[6] L. Amico, A. Osterloh, F. Plastina, R. Fazio, G. M. Palma, Phys. Rev. A. 69, 022304 (2004); quant-ph/0307048.

[7] J. Eisert, M. Cramer, Phys. Rev. A72, 042112 (2006); quant$p h / 0506250$.

[8] I. Peschel, J. Zhao, JSTAT P11002 (2005); quant-ph/0509002.

[9] R. Orús, J. I. Latorre, J. Eisert, M. Cramer, to appear in PRA (2006); quant-ph/0509023.

[10] M. Keyl, T. Matsui, D. Schlingemann, R. F. Werner; mathph/0604071.

[11] D. N. Page, Phys. Rev. Lett. 711291 (1993); hep-th/9306083. K. Życzkowski and H.-J. Sommers, J. Phys. A 34, 7111 (2001); quant-ph/0012101.

[12] B-Q. Jin and V. E. Korepin, J. Stat. Phys. 116, 79 (2004); quantph/0304108.

[13] P. Calabrese and J. Cardy, J. Stat.Mech. 0406 (2004) P002; hepth/0405152.

[14] M. M. Wolf, Phys. Rev. Lett. 96, 010404 (2006); quantph/0503219.

[15] D. Gioev and I. Klich, Phys. Rev. Lett. 96100503 (2006); quant-ph/0504151.

[16] T. Barthel, M. Chung, U. Schollwoeck; cond-mat/0602077.

[17] W. Li, L. Ding, R. Yu, T. Roscilde and S. Haas; quantph/0602094.

[18] D. Fursaev; hep-th/0602134.

[19] S. Ryu and T. Takayanagi; hep-th/0603001; hep-th/0605073

[20] R. Emparan; hep-th/0603081.
[21] F. Verstraete and J. I. Cirac, Phys. Rev. A70 060302(R) (2004); cond-mat/0407066.

[22] F. Verstraete, D. Porras and J. I. Cirac, Phys. Rev. Lett. 93, 227205 (2004); cond-mat/0404706.

[23] M. B. Plenio, J. Eisert, J. Dreissig and M. Cramer, Phys. Rev. Lett. 94, 060503 (2005); quant-ph/0405142.

M. Cramer, J. Eisert, M. B. Plenio and J. Dreissig, Phys. Rev. A 73, 012309 (2006); quant-ph/0505092.

[24] F. Verstraete and J. I. Cirac, Phys. Rev. B 73, 094423 (2006); cond-mat/0505140.

[25] M. B. Hastings, Phys. Rev. B 73, 085115 (2006); condmat/0508554.

[26] F. Vestraete, M. Wolf, D. Pérez-García and J. I. Cirac; quant$p h / 0601075$.

[27] F. Verstraete, J. I. Cirac, J. I. Latorre, E. Rico and M. M. Wolf, Phys.Rev.Lett. 94 (2005) 140601; quant-ph/0410227.

[28] K. Audenaert, J. Eisert, M.B. Plenio and R.F. Werner, Phys. Rev. A66, 042327 (2002); quant-ph/0205025.

[29] A. R. Its, B.-Q. Jin and V. E. Korepin, J. Phys. A 382975 (2005); quant-ph/0409027.

[30] I. Peschel, Journal of Statistical Mechanics (2004) P12005; cond-mat/0410416.

[31] L. Bombelli, R. K. Koul, J. Lee, R. D. Sorkin, Phys. Rev. D34 373 (1986).

[32] D. Kabat and M. J. Strassler Phys. Lett. B329,46 (1994); hepth/9401125.

[33] M. Cramer, J. Eisert, New J. Phys. 871 (2006); quantph/0509167.

[34] A. Botero, B. Reznik, Phys. Rev. A70 052329 (2004); quantph/0403233.

[35] A. Hamma, R. Ionicioiu and P. Zanardi, Phys. Rev. A71 022315 (2005); quant-ph/0409073.

[36] W. Dür, L. Hartmann, M. Hein, M. Lewenstein and H. Briegel, Phys. Lett. 94, 097203 (2005); quant-ph/0407075.

[37] J. I. Latorre and R. Orús, Phys. Rev. A 69, 062302 (2004); quant-ph/0308042. R. Orús and J. I. Latorre, Phys. Rev. A 69, 052308 (2004); quant-ph/0311017.

[38] M. C. Bañuls, R. Orús, J. I. Latorre, A. Pérez, P. Ruíz-Femenia, Phys. Rev. A 73022344 (2006); quant-ph/0503174. 
[39] J. I. Latorre, R. Orús, E. Rico and J. Vidal, Phys. Rev. A 71, 064101 (2005); cond-mat/0409611.

[40] J. Cardy and P. Calabrese, J.Stat.Mech. 0504 (2005) P010; cond-mat/0503393.

[41] G. De Chiara, S. Montangero, P. Calabrese, R. Fazio, J. Stat. Mech. (2006) P03001; cond-mat/0512586.

[42] F. Larsen and F. Wilczek, Ann. Phys. 243, 280 (1995); hepth/9408089.

[43] S.N.Solodukhin, Phys.Rev. D51 (1995) 609-617; hepth/9408089.

[44] D. Kabat, Nucl. Phys. B453, 281 (1995); hep-th/9503016.

[45] H. Casini, C. D. Fosco and M. Huerta, J. Stat. Mech. 0512 P012,2005; cond-mat/0505563.

[46] G. Vidal, Phys. Rev. Lett. 91, 147902 (2003); quantph/0301063; G. Vidal, Phys. Rev. Lett. 93, 040502 (2004); quant-ph/0310089.

[47] G. Vidal, cond-mat/0512165.

[48] J. I. Latorre, C. A. Lütken, E. Rico and G. Vidal, Phys. Rev. A71 (2005) 034301; quant-ph/0404120.

[49] R. Orús, Phys. Rev. A 71, 052327 (2005); quant-ph/0501110.

[50] A. B. Zamolodchikov, JETP Lett. 43 (1986) 730.

[51] A. Cappelli, D. Friedan and J. I. Latorre, Nucl. Phys. B352, 616(1991).

[52] S. Forte and J. I. Latorre, Nucl. Phys. B535, 709 (1998); hepth/9805015.

[53] H. Casini and M. Huerta, Phys. Lett. B600 142 (2004); hepth/0405111.

[54] H. Zhou, T. Barthel, J. Fjaerestad, U. Schollwoeck; condmat/0511732. 